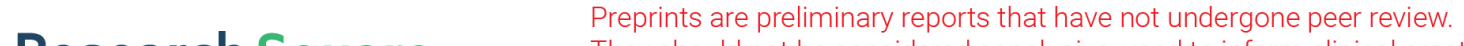 They should not be considered conclusive, used to inform clinical practice, or referenced by the media as validated information. \\ The Changes in The Utilization Rate of Traditional Chinese Medicine During the 2019 Coronavirus Pandemic
}

\section{Shun-Ku Lin}

National Yang-Ming University https://orcid.org/0000-0003-0067-2280

Chien-Tung Wu

National Yang-Ming Medical College: National Yang-Ming University

Chia-Jen Liu

National Yang-Ming Medical College: National Yang-Ming University

Hui-Jer Chou

Taipei City Hospital Renai Branch

Fu-Yang Ko

China Medical University

Ching-Hsuan Huang

China Medical University

Jung-Nien Lai ( $\sim$ ericlai111@gmail.com )

China Medical University, Taichung, Taiwan, ROC

\section{Research}

Keywords: COVID-19 pandemic, traditional Chinese medicine, utilization, mobile medicine

Posted Date: September 28th, 2020

DOI: https://doi.org/10.21203/rs.3.rs-77043/v1

License: (a) This work is licensed under a Creative Commons Attribution 4.0 International License. Read Full License 


\section{Abstract}

Background: Large-scale epidemics have changed people's medical behavior, and patients tend to delay non-urgent medical needs. However, the impact of the pandemic on the use of complementary and alternative medicine remains unknown.

Methods: This retrospective study aimed to analyze the changes in the number of traditional Chinese medicine (TCM) patients and examine the epidemic prevention policy during the coronavirus disease 2019 (COVID-19) pandemic. We analyzed the number of TCM patients in Taipei City Hospital from January 2017 to May 2020. We tallied the numbers of patients in each month and compared them with those in the same months last year. We calculated the percentage difference in the number of patients to reveal the impact of the COVID-19 pandemic on TCM utilization. We used the Mann-Whitney U test to examine whether there was a significant difference in the number of patients during the COVID-19 pandemic.

Results: We included a total of 41 months and 1,935,827 patients in this study. During the COVID-19 pandemic, the number of patients decreased significantly, except in February 2020. The numbers of patients during the COVID-19 pandemic had fallen by more than $15 \%$ compared with those in the same months last year. March and April had the greatest number of patient losses, with falls of $32.8 \%$ and $40 \%$, respectively. TCM patients declined significantly during the COVID-19 pandemic, and mobile medicine provided to rural areas fell considerably. Among all the TCM specialties, pediatrics and traumatology, as well as infertility treatment, witnessed the most significant decline in the number of patients. However, the number of cancer patients has reportedly increased.

Conclusions: The COVID-19 pandemic decreased the utilization rate of TCM, especially for mobile healthcare in rural areas. We suggest that the government pay attention to the medical disparity between urban and rural areas, which are affected by the pandemic, as well as allocate adequate resources in areas deprived of medical care.

\section{Introduction}

Large-scale epidemics have changed the people's medical behavior and medical utilization ${ }^{1}$.

During the Severe Acute Respiratory Syndrome (SARS) epidemic in 2003, Taiwan's medical utilization rate and medical expenses dropped remarkably. Patients tended to delay non-urgent medical needs and postpone elective procedures ${ }^{2}$. For medical needs that could not be deferred, such as childbirth, patients receive medical treatment in small local hospitals, and the length of hospital stay was notably reduced ${ }^{3}$. During a pandemic, resources for medically disadvantaged patients, such as patients in rural areas and those receiving palliative care, will also be limited ${ }^{4}, 5$. However, the use of traditional Chinese medicine (TCM) during SARS marginally increased in Taiwan. This could be attributed to people choosing complementary and alternative medicine as a substitute for Western medicine. The mode of transmission 
of coronavirus disease 2019 (COVID-19) in Taiwan is different from that of SARS. COVID-19 was transmitted from abroad with limited community transmission, while SARS was mainly transmitted via nosocomial infection. The impact of the current pandemic on the use of complementary and alternative medicine remains unknown. This study aimed to analyze the changes in the number of TCM patients and examine the epidemic prevention policies during the COVID-19 pandemic.

\section{Materials And Methods}

This retrospective survey aimed to analyze the difference in the number of TCM patients and examine the epidemic prevention policy during the COVID-19 pandemic. The Taipei City Hospital's Research Ethics Committee reviewed and approved this study (review number TCHIRB-10904002-E). We evaluated the number of TCM patients in Taipei City Hospital from January 2017 to May 2020, including outpatient and ward consultations. Taipei City Hospital consists of ten hospitals and is the largest public hospital in Northern Taiwan. Medical services cater to the entire city, spanning 271.8 square kilometers with 2.6 million residents. The hospital provides TCM services, including outpatient services and ward consultations. It also offers specialized medical services in TCM such as internal medicine, acupuncture, Pediatrics, obstetrics and gynecologyand traumatology. Moreover, Taipei City Hospital also provides mobile medical services for rural areas that lack medical resources. Their TCM medical team visits rural clinics weekly. Since Taipei City Hospital has a complete medical system and serves all people in Taipei City, it is suitable as a representative sample for the evaluation of medical utilization. ${ }^{6}$

We obtained the number of TCM patients from the hospital information system, including all departments. We computed the number of patients each month and compared it with the same months last year. We divided the patients by the following specialties: internal medicine, acupuncture, traumatology, obstetrics and gynecology, and pediatrics. Furthermore, we separately analyzed the number of stroke, cancer, and infertility patients. Taiwan Health Insurance encourages TCM practitioners to provide medical services for patients with the aforementioned diseases and to increase medical claims. We also scrutinized the number of mobile medical visits in rural areas, including partial townships and outlying islands.

We calculated the percentage difference in the number of patients to reveal the impact of the COVID-19 pandemic on TCM usage. We used three control groups as a benchmark to understand the impact of the pandemic on the number of TCM patients. First, we used the number of patients in Taipei City Hospital in the same months of the previous year (January to May 2019) to compare to the number of patients during the COVID-19 pandemic. Second, we employed the Mann-Whitney U test to examine whether there was a significant disparity between the number of patients during the COVID-19 pandemic and that in the same months in the past five years (January to May 2015 to 2019). Third, we used the variation in the number of TCM patients nationwide during the SARS epidemic as the control group to compare the difference in the number of patients during COVID-19. We screened TCM users from Taiwan's National Health Insurance Research Database and calculated the number of TCM doctors in the country and the 
percentage difference. We used MedCalc Statistical Software Version 19.4.0 (MedCalc Software Ltd, Ostend, Belgium) to analyze the data.

\section{Results}

The study included a total of 1,935,827 patients who are recipients of the services of Taipei City Hospital. Figure 1 shows the number of patients and the percentage variation during the COVID-19 pandemic. The number of patients declined significantly in the present year, except in February 2020. The number of patients has fallen by more than $15 \%$ compared last year. March and April had the substantial number of losses, falling by $32.8 \%$ and $40 \%$, respectively.

We compared the number of TCM patients during the SARS epidemic and the COVID-19 pandemic in Figure 2. We used the main epidemic period of the disease in Taiwan as the comparison standard. The epidemic period of SARS ran from February to July 2003 in Taiwan, and COVID-19 was mainly from January to May 2020. The number of TCM patients increased marginally during the SARS epidemic. On the contrary, the number of patients with TCM dropped significantly during COVID-19.

We separately analyzed the number of patients in different TCM specialties and presented the percentage change as compared to the previous year in Figure 3. Pediatrics and traumatology received the most significant declines, with the greatest reductions exceeding 50\%. Acupuncture and obstetrics and gynecology also fell by more than $40 \%$, compared to the same months last year. Internal medicine had a relatively stable number of patients, with a decrease of only $26.8 \%$ at most.

Figure 4 shows the percentage change in the number of patients in different TCM treatment programs. The number of infertility treatments declined during the COVID-19 pandemic. As for the sum of cancer patients, on the contrary, it revealed an upward trend. The number of stroke patients treated with TCM has also decreased, but it was not pronounced as compared to other diseases. Moreover, the number of patients utilizing mobile medicine in rural areas has fallen sharply during the pandemic, falling by more than 60\% from March to May 2020.

We employed the Mann-Whitney $U$ test to examine whether the number of TCM patients declined significantly during the COVID-19 pandemic. It showed that the total number of patients and the number of mobile medical patients decreased significantly, with a p-value of less than 0.05 . The patients of TCM specialists declined considerably as well, including internal medicine, acupuncture, traumatology, obstetrics and gynecology, and pediatrics. On the other hand, there was no significant difference in the number of patients with specific diseases such as stroke and cancer. 
Table 1.

Significant changes in the number of patients in TCM before and during the COVID-19 pandemic using the Mann-Whitney U test.

\begin{tabular}{|llllll|}
\hline & $\begin{array}{l}\text { Average rank of } \\
\text { first group }\end{array}$ & $\begin{array}{l}\text { Average rank of } \\
\text { second group }\end{array}$ & $\begin{array}{l}\text { Mann- } \\
\text { Whitney U }\end{array}$ & $\begin{array}{l}\text { Test } \\
\text { statistic } \\
\text { Z }\end{array}$ & $\begin{array}{l}\text { Two-tailed } \\
\text { probability }\end{array}$ \\
\hline Total & 23.306 & 4.4 & 7 & 3.309 & $0.0009^{*}$ \\
\hline $\begin{array}{l}\text { Internal } \\
\text { medicine }\end{array}$ & 23.250 & 4.8 & 9 & 3.227 & $0.0013^{*}$ \\
\hline Acupuncture & 23.120 & 5.0 & 11 & 3.118 & $0.0018^{*}$ \\
\hline Traumatology & 23.167 & 5.4 & 12 & 3.108 & $0.0019^{*}$ \\
\hline $\begin{array}{l}\text { Obstetrics and } \\
\text { Gynecology }\end{array}$ & 23.306 & 4.4 & 7 & 3.307 & $0.0009^{*}$ \\
\hline Pediatrics & 23.361 & 4.0 & 5 & 3.387 & $0.0007^{*}$ \\
\hline Mobile medicine & 23.500 & 3.0 & 0 & 3.586 & $0.0003^{*}$ \\
\hline Stroke & 22.250 & 12.0 & 45 & 1.793 & 0.0730 \\
\hline Cancer & 19.806 & 29.6 & 47 & -1.714 & 0.0866 \\
\hline
\end{tabular}

\section{Discussion}

This study revealed that the quantity of TCM patients declined significantly during the COVID-19 pandemic, and mobile medicine provided to rural areas dropped even further. Patients availing TCM specialty services have also decreased, with pediatrics and traumatology having the most significant decline, as well as infertility treatment. However, the number of cancer patients has notably increased.

There was a significant difference in the utilization rate of TCM treatments among Taiwanese patients during SARS and the COVID-19 pandemic. During the SARS epidemic in 2003, the utilization of TCM services increased. Conversely, during the COVID-19 pandemic, the utilization rate of TCM decreased markedly. We surmise that the Taiwanese government used the same strategy during SARS in 2003 and used the national network information system and health system to combat the COVID-19 pandemic. ${ }^{7}$

The SARS epidemic had spread to more than 30 countries, affecting 8,096 people, resulting in 774 deaths worldwide from November 1, 2002 to July 31, 2003. Using the approach during the SARS outbreak, in January, the Taiwanese government issued the COVID-19 case definition and notification process, requiring all physicians (including TCM) to report suspected cases during the outset of COVID-19 in Wuhan. Due to the enormous losses to society because of SARS and considering that COVID-19 can be asymptomatic and is highly infectious, the Taiwanese government implemented stricter restrictions during this pandemic. The subsequent epidemic policy for COVID-19 includes maintaining social 
distance, wearing of face masks in public, and prohibition of gatherings of more than 50 people in a meeting or conference. Therefore, the utilization rate of TCM dropped by more than $30 \%$ after March 2020. It is common knowledge that the COVID-19 has spread to more than 188 countries, affecting 14.03 million people, resulting in 602,000 deaths worldwide within only six months. Fortunately, compared to the same period in 2003 , the proportion of probable SARS cases dropped from $8.2 \%$ (664 Taiwanese cases per 8096 global cases) to $0.0032 \%$ ( 455 Taiwanese cases per 14,288,898 global cases) in 2020 in Taiwan.

The number of people quarantined at home was 10,904 in the 2003 SARS epidemic and 77,948 in the 2020 COVID-19 pandemic. During COVID-19, the number of people quarantined at home exceeds threethousandths of the total population, and they are required to seek medical treatment only at designated medical centers. The decline in Chinese medicine utilization can be attributed to the stringent health policies especially for people in quarantine who are only allowed access to medical care at designated health facilities.

Notably, we found that the number of mobile medical services provided to rural areas dropped significantly. Rural areas lack medical resources, and people's health status is worse than that in urban areas. Taiwan's Ministry of Health and Welfare started TCM mobile medical services in rural areas in 2004 and provides home medical care for bedridden patients.

However, the present study postulates that a large-scale epidemic of infectious diseases will consume a large amount of medical resources in urban areas and reduce medical accessibility in rural areas. We suggest that the government allocate adequate funds and provide medical workforce to maintain access to medical services and institute fairness in terms of resource allocation to rural areas, especially during the pandemic.

Surprisingly, it is peculiar that the number of TCM visits from cancer patients increased from $43.8 \%$ to $85.2 \%$ when compared with that in the same months last year. Clinical studies have found that TCM participation in cancer treatment can improve the quality of life and reinforce the effects of chemotherapy and radiotherapy ${ }^{8,9}$. In addition, TCM treatment can increase the survival time of cancer patients and reduce overall medical costs. ${ }^{10,11}$ Cancer patients with or without cancer-related treatment are definitely vulnerable populations as they are immunocompromised. We speculate that because TCM is deeply ingrained into Taiwan's cancer treatment and is expected to improve or boost immune function, the TCM medical needs for cancer patients, therefore, increased even during the COVID-19 pandemic.

\section{Conclusion}

Preemptive preparedness to fight COVID-19 is paramount. The COVID-19 pandemic and restrictive health policies decreased the TCM utilization rate, especially for mobile healthcare in rural areas. We therefore conclude that there should be adequate fund allocation for the healthcare system and that TCM medical 
workforce for cancer patients should be provided to maintain medical access and impartiality in terms of resource allocation in rural areas, especially during the pandemic.

\section{Abbreviations}

TCM traditional Chinese medicine

COVID-19 coronavirus disease 2019

SARS Severe Acute Respiratory Syndrome

\section{Declarations}

\section{Ethics approval and consent to participate}

The Taipei City Hospital's Research Ethics Committee reviewed and approved this study (review number TCHIRB-10904002-E)

\section{Consent for publication}

All coauthors have reviewed and approved of the manuscript prior to submission.

\section{Availability of data and materials}

The datasets used and/or analyzed during the current study are available from the corresponding author on reasonable request.

\section{Competing interests}

The authors declare no competing interests.

\section{Funding}

This research was funded by the Department of Health, Taipei City Government, Research Program, grant numbers 10901-62-003 and 10801-62-001, and supported by Department of Chinese Medicine and Pharmacy, Ministry of Health and Welfare, grant numbers MOHW-109-CMB-03.

\section{Authors' contributions}


Shun-Ku Lin and Jung-Nien Lai planned and designed the research protocol. Chien-Tung Wu and Hui-Jer Chou assisted in research and data analysis. Chia-Jen Liu, Fu-Yang Ko, and Ching-Hsuan Huang provided professional advice in writing the paper. All coauthors have reviewed and approved of the manuscript prior to submission.

\section{Acknowledgments}

We thank the Taipei City Hospital and the Department of Traditional Chinese Medicine of the Ministry of Health and Welfare for assisting in the research.

\section{References}

1. Lu, T. H.; Chou, Y. J.; Liou, C. S., Impact of SARS on healthcare utilization by disease categories: implications for delivery of healthcare services. Health Policy 2007, 83 (2-3), 375-81.

2. Chang, H. J.; Huang, N.; Lee, C. H.; Hsu, Y. J.; Hsieh, C. J.; Chou, Y. J., The impact of the SARS epidemic on the utilization of medical services: SARS and the fear of SARS. Am J Public Health 2004, 94 (4), 562-4.

3. Lee, C. H.; Huang, N.; Chang, H. J.; Hsu, Y. J.; Wang, M. C.; Chou, Y. J., The immediate effects of the severe acute respiratory syndrome (SARS) epidemic on childbirth in Taiwan. BMC Public Health 2005, 5, 30 .

4. Chen, T. J.; Lin, M. H.; Chou, L. F.; Hwang, S. J., Hospice utilization during the SARS outbreak in Taiwan. BMC Health Serv Res 2006, 6, 94.

5. Fisk, M.; Livingstone, A.; Pit, S. W., Telehealth in the Context of COVID-19: Changing Perspectives in Australia, the United Kingdom, and the United States. J Med Internet Res 2020, 22 (6), e19264.

6. Chou, Y. C.; Yen, Y. F.; Feng, R. C.; Wu, M. P.; Lee, Y. L.; Chu, D.; Huang, S. J.; Curtis, J. R.; Hu, H. Y., Impact of the COVID-19 Pandemic on the Utilization of Hospice Care Services: A Cohort Study in Taiwan. J Pain Symptom Manage 2020.

7. Lai, C. C.; Wang, C. Y.; Wang, Y. H.; Hsueh, S. C.; Ko, W. C.; Hsueh, P. R., Global epidemiology of coronavirus disease 2019 (COVID-19): disease incidence, daily cumulative index, mortality, and their association with country healthcare resources and economic status. Int $J$ Antimicrob Agents 2020, 55(4), 105946.

8. Wang, C.; Yang, M.; Fan, Y.; Pei, X., Moxibustion as a therapy for breast cancer-related lymphedema in female adults: a preliminary randomized controlled trial. Integr Cancer Ther 2019, 18, 1534735419866919.

9. Lu, Y.; Qu, H. Q.; Chen, F. Y.; Li, X. T.; Cai, L.; Chen, S.; Sun, Y. Y., Effect of Baduanjin Qigong exercise on cancer-related fatigue in patients with colorectal cancer undergoing chemotherapy: a randomized controlled trial. Oncol Res Treat 2019, 42 (9), 431-439. 
10. Lin, P. H.; Lin, S. K.; Hsu, R. J.; Pang, S. T.; Chuang, C. K.; Chang, Y. H.; Liu, J. M., Spirit-quieting traditional Chinese medicine may improve survival in prostate cancer patients with depression. $J$ Clin Med 2019, 8 (2).

11. Liu, J. M.; Lin, P. H.; Hsu, R. J.; Chang, Y. H.; Cheng, K. C.; Pang, S. T.; Lin, S. K., Complementary traditional Chinese medicine therapy improves survival in patients with metastatic prostate cancer. Medicine (Baltimore) 2016, 95 (31), e4475.

\section{Figures}
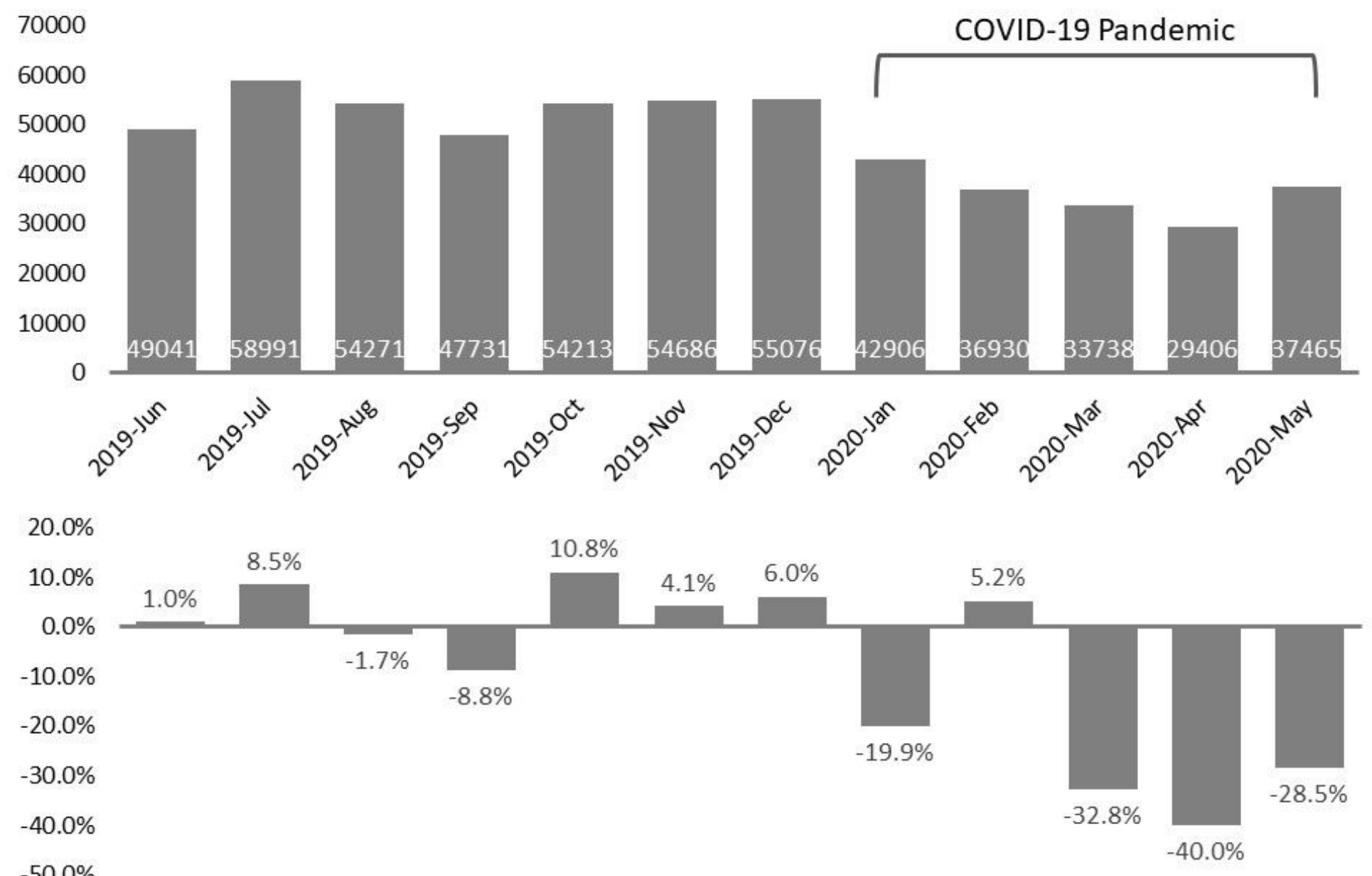

$-50.0 \%$
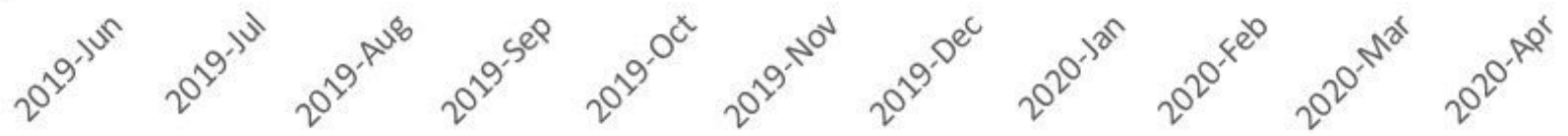

\section{Figure 1}

Changes in the number of TCM patients during COVID-19 pandemic. We recorded the number of TCM patients in Taipei City Hospital from June 2019 to May 2020, and the percentage difference in comparison to that in the same months of the previous year. Since the COVID-19 pandemic began to spread worldwide in January 2020, the number of Chinese medicine clinics has dropped significantly, and it has fallen by more than $15 \%$ compared to that in the same months last year. 
- COVID-19 ISARS

$20.0 \%$

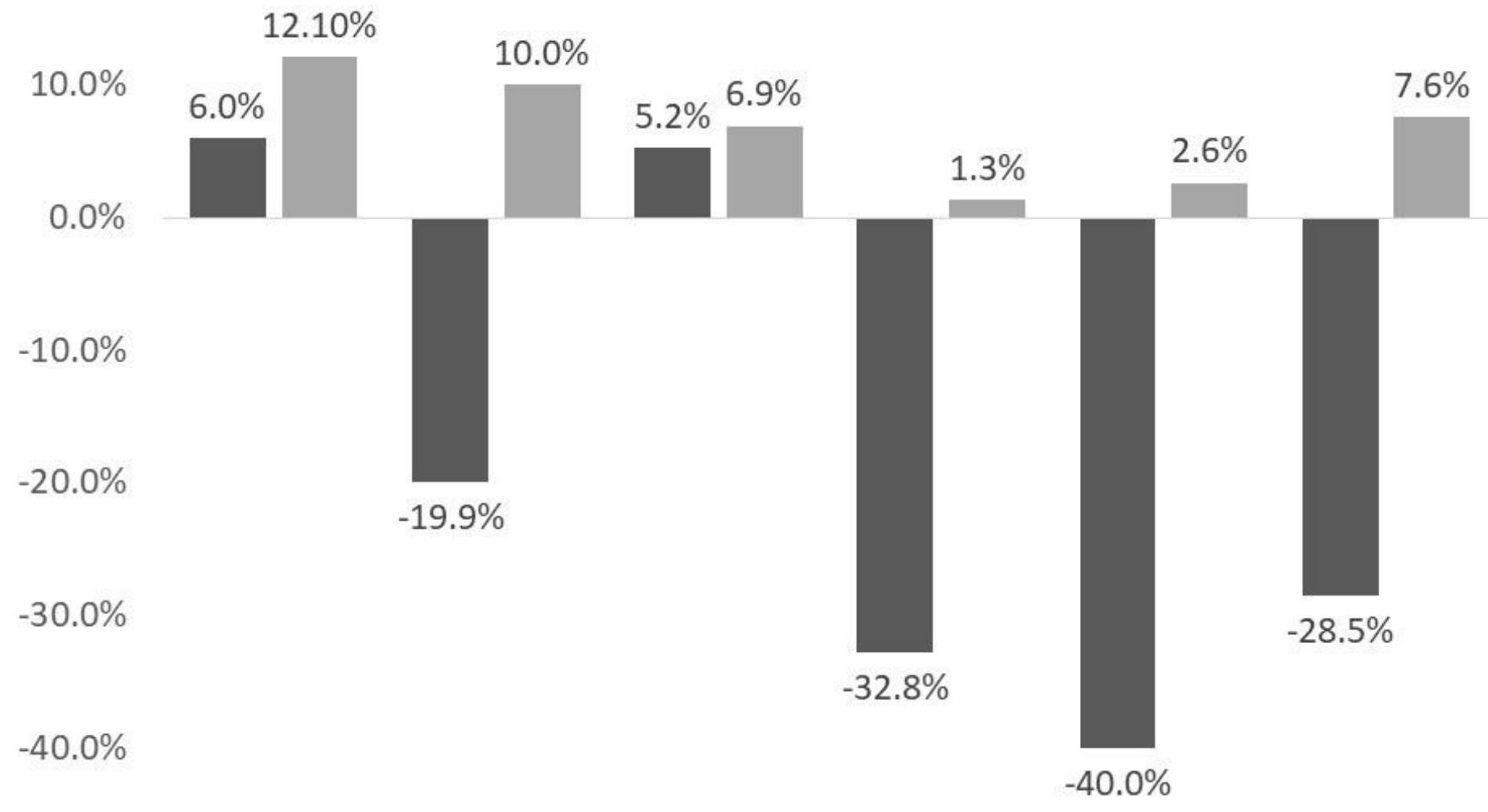

$-50.0 \%$

Before $\quad 1$ month 2 month 3 month 4 month 5 month

Figure 2

A comparison of the number of TCM patients during the SARS epidemic and the COVID-19 pandemic. The number of TCM patients increased marginally during the SARS epidemic. While, the number of patients utilizing TCM dropped significantly during COVID-19. 


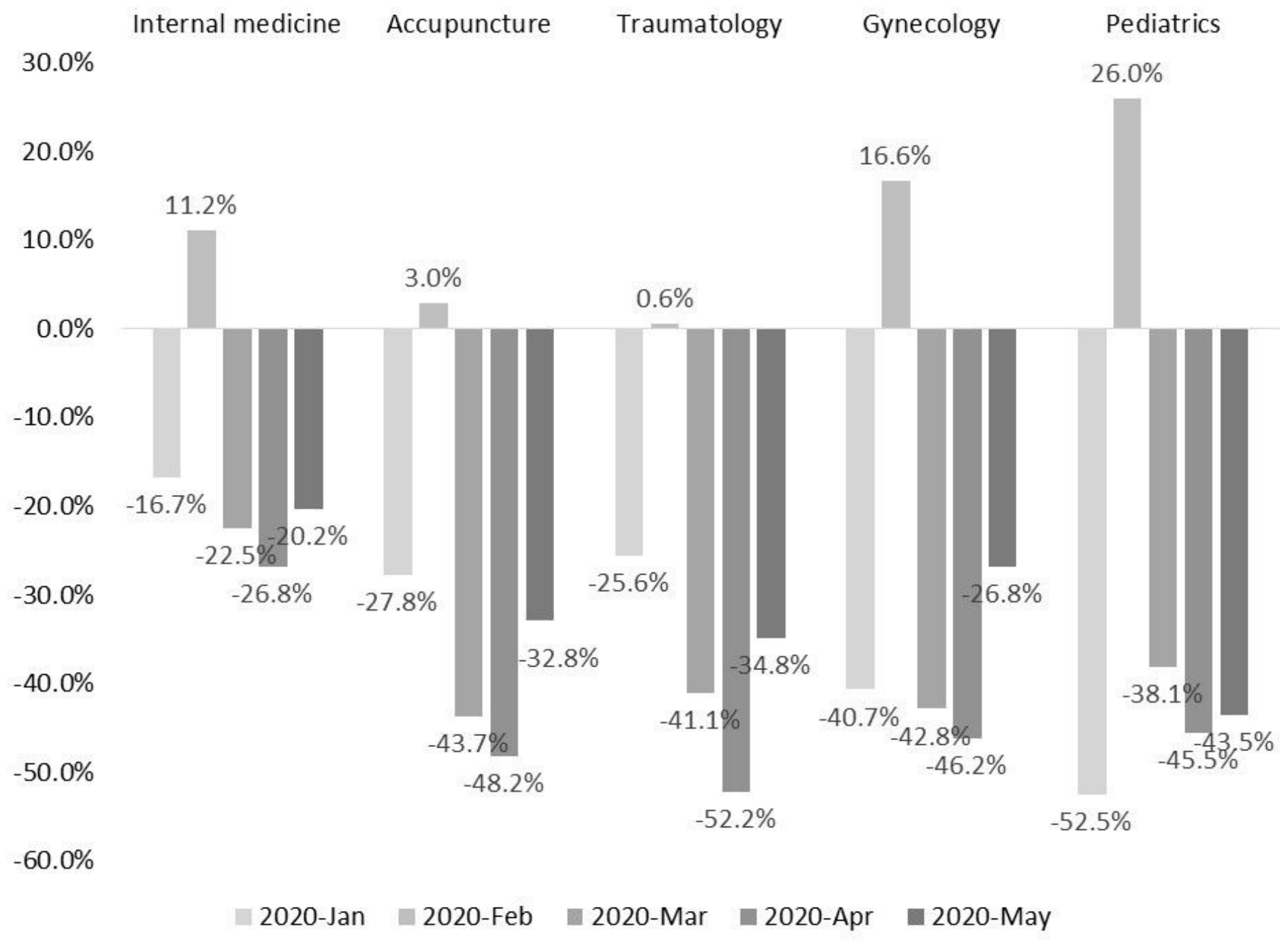

Figure 3

The percentage difference in the number of patients in each specialty in comparison to that in the same months last year. Pediatrics and traumatology received the most significant declines, with the most reductions exceeding $50 \%$. Acupuncture and obstetrics and gynecology also fell by more than $40 \%$, compared to the same months last year. 


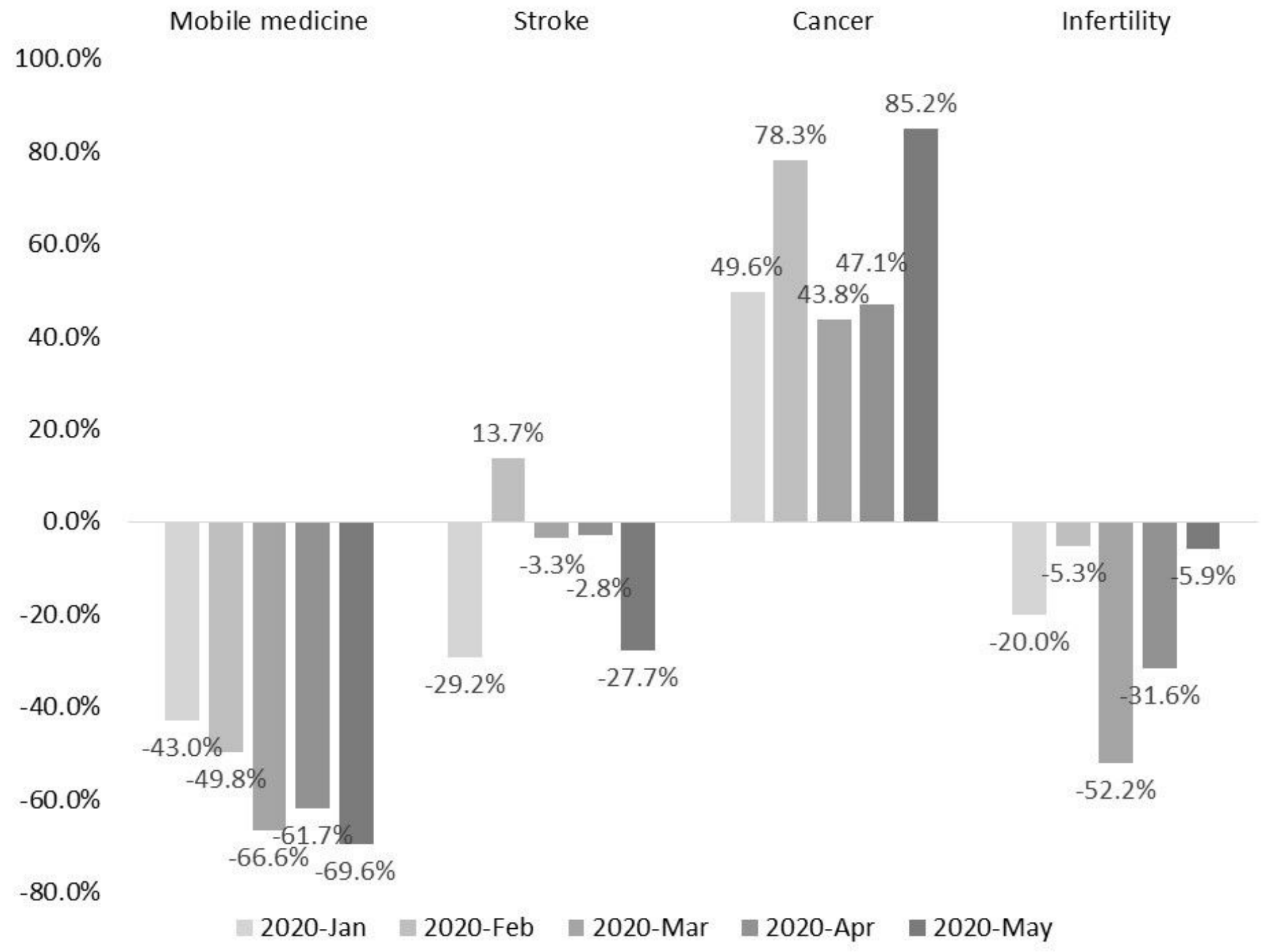

\section{Figure 4}

The percentage difference in the number of patients in different TCM treatment programs. During the COVID-19 pandemic, mobile TCM services and infertility treatments in rural areas declined significantly. On the contrary, the number of cancer patients receiving adjuvant therapy with TCM increased significantly as compared with that in the same months last year. 